Instructions for authors, subscriptions and further details:

\title{
La Descentralización de la Formación para el Empleo. Las Implicaciones de los Agentes Participantes en los Programas Formativos para Desempleados/as en Andalucía
}

María Rosario Carvajal Muñoz ${ }^{1}$

1) Universidad de Cádiz, Spain

Date of publication: June $25^{\text {th }}, 2015$

Edition period: June 2015-October 2015

To cite this article: Carvajal Muñoz, M.R. (2015). La Descentralización de la Formación para el Empleo. Las Implicaciones de los Agentes Participantes en los Programas Formativos para Desempleados/as en Andalucía. International Journal of Sociology of Education, 4(2), 101- 127. doi: 10.17583/rise.2015.1493

To link this article: http://dx.doi.org/10.17583/rise.2015.1493

PLEASE SCROLL DOWN FOR ARTICLE

The terms and conditions of use are related to the Open Journal System and to Creative Commons Attribution License (CC-BY) 



\section{The Decentralization of the Training for Employment. The Implications of the Participating Agents in the Training Programs for Unemployed People in Andalusia}

María Rosario Carvajal Muñoz

University of Cádiz

(Received: 7 April 2015; Accepted: 8 June 2015; Published: 25 June 2015)

\section{Abstract}

This text analyses the local entities and the students who are involved in these training programs of occupational training courses and Expertise Workshop, Trade House and Workshop on Employment in Andalusia. This data was collected on the web page of the autonomic government, contrasting with the information obtained from case research about two Sevillian towns, where was done in-depth interviews, as well. The results point out contingent aspects that the establishment of these programs brings for the concrete peculiarities of the territory, depending on the typology participating local entities. But also because of the design of these programs determines the scope of action of the local entities. Additionally, it reflects, according to this policy, on the decline of the labour identity as a characteristic of the salary society to a social construction of new identity criterions which are further individual and heterogeneous

Keywords: collaborator centres, councils, public enterprises, private enterprises, non-governmental organisations, typology of unemployeds people. 


\section{La Descentralización de la Formación para el Empleo. Las Implicaciones de los Agentes Participantes en los Programas Formativos para Desempleados/as en Andalucía}

María Rosario Carvajal Muñoz

Universidad de Cádiz

(Recibido: 7 de Abril 2015; Aceptado: 8 de Junio 2015; Publicado: 25 Junio 2015)

\section{Resumen}

Este texto analiza a los agentes locales y al alumnado participantes en los programas formativos de cursos de formación y de Escuelas Taller, Casas de Oficios y Talleres de Empleo en Andalucía. Los datos se recabaron de la página de Internet del gobierno autonómico, contrastando con la información obtenida en una investigación de casos en dos municipios sevillanos, utilizando también entrevistas en profundidad. Los resultados apuntan a aspectos contingentes que traen consigo la implantación de estos programas, por las particularidades concretas del territorio, según la tipología de entidades locales. Pero también porque el diseño de estos programas condiciona el margen de maniobra de las entidades locales. Asimismo, se reflexiona a partir de esta política sobre el declive de la identidad laboral característica de la sociedad salarial, y la construcción social de nuevos criterios identitarios, más individualistas y heterogéneos

Palabras clave: centros colaboradores, ayuntamientos, empresas públicas, empresas privadas, organizaciones no gubernamentales, colectivos de desempleados/as. 



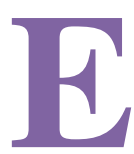

1 presente trabajo se inicia con cuestiones relativas a las características de la participación de los desempleados/as y de los agentes locales que participan en las modalidades formativas de cursos de formación profesional ocupacional, y en los programas formativos de Escuelas Taller (ET), Casas de Oficios (CO) y Talleres de Empleo (TE), estos programas arrancan en la década de los ochenta por iniciativa del gobierno central español ${ }^{1}$. El análisis en torno a la participación de los desempleados/as y agentes locales en estas dos modalidades formativas se relaciona con la crisis de la sociedad salarial frente a los elementos característicos de un nuevo orden social (Moral, 2007, p.1272), que se asienta en un modelo organizativo más descentralizado.

Justamente, la política de formación para desempleados/as tiene su origen con la crisis de la sociedad salarial en la década de los ochenta del siglo pasado (Moral, 2007, Alonso, 2000), y en este contexto las acciones formativas se imparten con la condición necesaria, aunque no suficiente, de que contribuyan a la posterior incorporación del alumnado en el mercado de trabajo. Pero por otro lado, se tratan de programas formativos asentados en el territorio, que priorizan la participación de agentes locales no lucrativos, públicos y privados en la implementación de estas acciones, y dirigidas a colectivos de desempleados muy heterogéneos. Este protagonismo de los agentes locales estuvo promovido inicialmente por la OCDE y la Unión Europea, de modo que la descentralización de las políticas de empleo se ha impuesto como la organización más eficaz en la implementación de acciones para combatir el desempleo en muchos otros países (Caswell, et al., 2010, p.384. Martínez Lucio et al., 2007).

Marinetto (2003, p.109) considera que las políticas de formación implican un tipo particular de moralidad personal y forma positiva de vida para la comunidad. Por su parte, la política de formación combina los valores propios de la sociedad salarial, focalizada en la inserción laboral, promovida por estos programas de formación, junto con el reconocimiento de la necesidad de que la formación sirva para la promoción integral de la persona, que contribuya a su bienestar dentro de la comunidad. Asimismo, los agentes locales mantienen comportamientos peculiares según características del territorio. Algunas evaluaciones realizadas en la década de los noventa ya mostraban que el impacto de los programas de servicios sociales para el empleo estaba siendo altamente contingente dependiendo de 


\section{Carvajal Muñoz - Formación para el Empleo}

las condiciones de los mercados de trabajo locales y de la capacidad de agentes sociales y redes de contactos para dar resultados eficientes (Hutchinson \& Cambell, 1998). En un estudio más reciente, Marston, McDonald y Wright (2011) analizaron el papel de las organizaciones no lucrativas en la implementación de programas de servicios sociales y de empleo en Inglaterra y Austria, y los resultados apuntaban a comportamientos contingentes entre las organizaciones locales participantes en el ámbito comunitario.

En lo que respecta a este trabajo sobre la política de formación en Andalucía se ha realizado principalmente gracias a la información recabada en Internet que recoge la página Web de la Junta de Andalucía, tanto en lo relativo a los cursos de formación como para los programas de ET, CO y TE. Pero también se han tenido en cuenta los textos normativos que regulan estos programas formativos, además de contrastarse la información recabada con la recogida en una investigación realizada en Osuna y Estepa, provincia de Sevilla, utilizando principalmente entrevistas en profundidad ${ }^{2}$. Los resultados indican que tanto en la modalidad formativa de cursos de formación, como en la de ET, CO y TE, participan entidades locales sin ánimo de lucro en la implementación de estas acciones, pero también empresas privadas, empresas públicas y ayuntamientos. En la implementación de cursos de formación se han beneficiados colectivos diversos de desempleados/as, desde mujeres, a inmigrantes, colectivos pertenecientes a grupos étnicos, jóvenes, ex presidiarios y discapacitados; aunque el mayor porcentaje del alumnado de estos cursos pertenece a población desempleada en general, sin determinar grupo específico. Es diferente en la modalidad formativa de ET, CO y TE, pues al estar diseñados para personas con fracaso escolar, o en particular situación de vulnerabilidad social, da entrada en mayor proporción a las personas desempleadas con mayores riesgos de exclusión.

En cuanto a las entidades promotoras de los proyectos son los ayuntamientos los que mayoritariamente participan en los programas de ET, $\mathrm{CO}$ y TE, seguidos de empresas públicas, y en menor medida entidades no lucrativas. En cualquier modo, estos resultados apuntan a comportamientos contingentes entre territorios. Esta contingencia se argumenta en base a la confrontación de los datos recabados para el conjunto andaluz contrastando con los aspectos teóricos que justifican y marcan los objetivos de estas dos 
modalidades formativas, ya que las realidades territoriales responden a dinámicas internas según características de las entidades locales presentes en cada territorio, pero también el propio diseño de la política de formación repercute en el tipo de implicación de las entidades locales en estas acciones formativas. En este texto, algunos de los aspectos contingentes se manifiestan también al confrontar con las particularidades de ambas formaciones en los dos municipios sevillanos estudiados, por diferencias significativas entre ellos y con respecto al conjunto andaluz, principalmente en lo relativo a las entidades locales participantes, y para el programa de cursos de formación.

\section{Alumnado y Entidades Locales Participantes en los Cursos de Formación}

La modalidad de cursos de formación de la que trata esta sección comenzó su andadura en 1985, bajo la responsabilidad del Instituto Nacional de Empleo. En sus inicios esta formación iba dirigida a colectivos con especiales dificultades de inserción laboral, haciendo particular hincapié en parados de larga duración, menores de 25 años, mujeres, y colectivos de desempleados/as con necesidades formativas para la reconversión industrial. Como reconoce Pérez-Díaz y Rodríguez (2002), el actual programa está impartido por centros colaboradores públicos y privados. El cariz formativo de este programa es bien distinto al que se impartió hasta bien entrada la década de los setenta, ya que se trataba de una formación profesional de adultos para satisfacer necesidades formativas en contexto de desarrollo económico, proporcionando una formación específica muy circunscrita a las características de los puestos de trabajo existentes (Pérez- Díaz y Rodríguez, 2002, p.45).

En la década de los noventa del siglo XX se da la descentralización de este programa formativo con el traspaso de competencias de la administración central a la Junta de Andalucía ${ }^{3}$. Esta descentralización ha propiciado la participación de un gran número de entidades locales en la implementación de esta modalidad formativa. La tabla 1 recoge la evolución en el número de centros colaboradores ${ }^{4}$ en las ocho provincias andaluzas de 2011 a 2015, apreciándose un descenso en el número de centros colaboradores en las provincias orientales de Granada y Jaén, en tanto que 


\section{Carvajal Muñoz - Formación para el Empleo}

en las demás se sigue manteniendo el incremento de centros colaboradores en el transcurso de estos cuatro años ${ }^{5}$. Los monográficos consultados ${ }^{6}$ de 2005 y 2009, publicados por la Junta de Andalucía, distinguen entre entidades sin ánimo de lucro, entidades privadas, entidades públicas, y corporaciones locales, y muestran cómo la participación proporcional en la impartición de cursos de estas entidades se mantuvo la misma en ambos años ${ }^{7}$. En cambio, comparando estas cifras con el estudio realizado en los municipios sevillanos de Osuna y Estepa se observan diferencias que destacan aspectos contingentes en cuanto a la tipología de entidades participantes (Carvajal, 2014) con respecto al conjunto andaluz para 2005 y 2009. Las corporaciones locales y las entidades públicas son las que más cursos impartieron en estos dos municipios, muy por encima del 50\%, frente al $13 \%$ en el conjunto andaluz, y dándose una participación muy pequeña de las entidades privadas y sin ánimo de lucro $^{8}$ (Carvajal, 2002, 2014). En este sentido, Finn (2000, p.43) destaca que las evaluaciones realizadas sobre la implicación de los agentes locales en los programas de políticas de empleo desarrollados en la comunidad llegan a resultados contingentes, porque los mercados de trabajo locales, y la capacidad de los agentes locales para hacer frente a estos nuevos problemas sociales, están dando resultados diferentes según los territorios. De hecho, en Osuna, a diferencia de Estepa, hubo mayor implicación de los sindicatos locales, y de la asociación de personas con discapacidad física, como se verá más adelante, en la impartición de estos cursos de formación. Por tanto, los aspectos contingentes se aprecian también comparando ambos municipios. 
Tabla 1

Centros Colaboradores en Andalucía de 2011 a 2015

\begin{tabular}{lccc}
\hline & 2011 & 2012 & 2015 \\
\hline Granada & 1.128 & 939 & 1014 \\
Sevilla & 1.919 & 2004 & 2129 \\
Málaga & 1.476 & 1572 & 1666 \\
Cádiz & 1.151 & 1.175 & 1284 \\
Huelva & 926 & 948 & 983 \\
Córdoba & 1.565 & 1.593 & 1642 \\
Jaén & 1.125 & 1.094 & 1073 \\
Almería & 605 & 632 & 680 \\
Total & 9.895 & 9.957 & 10.471 \\
\hline
\end{tabular}

Elaboración propia a partir de la información obtenida en Internet: http://www.juntadeandalucia.es/empleo/macenco/avanzada.jsp

Por otro lado, en cuanto a las especialidades formativas ${ }^{9}$ más homologadas por los centros colaboradores destacan las que recogen la tabla 2, mostrando una concentración de centros homologados en tres especialidades formativas, Administración y Gestión, Informática y Comunicaciones y Servicios Socioculturales y a la Comunidad, ya que están presentes en aproximadamente una cuarta parte del total de centros colaboradores andaluces. El resto de especialidades formativas no contempladas en la tabla, 23 especialidades de las consultadas ${ }^{10}$, han sido homologadas en menos de un $9 \%$ de los centros. En el estudio realizado en Osuna y Estepa los centros colaboradores ofertaban las especialidades formativas que más se adecuaran a los intereses de su entidad y a las demandas formativas que les hacían los desempleados/as, en particular para centros colaboradores de ayuntamientos, y en mucha menor medida para responder a las necesidades del mercado de trabajo local (Carvajal, 2002, 2014). Y es que la participación de las entidades locales en estas acciones formativas parecen responder más a motivaciones particulares. Probablemente, una de las principales debilidades de estos programas formativos sea la ausencia de un compromiso más fuerte con la creación de empleo, especialmente en las zonas con más altas tasas de paro (Peck, 1998, Turok \& Webster, 1998). 
Tabla 2

Centros Colaboradores por familias formativas 2015

\begin{tabular}{l|ccccc}
\hline & $\begin{array}{l}\text { ADG } \\
\text { Administración } \\
\text { y Gestión }\end{array}$ & $\begin{array}{l}\text { SSC Servicios } \\
\text { Socioculturales } \\
\text { y la } \\
\text { Comunidad }\end{array}$ & $\begin{array}{l}\text { IFC Informática } \\
\text { Comunicaciones }\end{array}$ & $\begin{array}{l}\text { SAN } \\
\text { Sanidad }\end{array}$ & $\begin{array}{c}\text { Total de } \\
\text { centros } \\
\text { colaboradores }\end{array}$ \\
\hline Granada & 362 & 288 & 252 & 129 & 1014 \\
Sevilla & 617 & 557 & 516 & 251 & 2129 \\
Málaga & 459 & 374 & 374 & 149 & 1666 \\
Cádiz & 366 & 301 & 297 & 165 & 1284 \\
Huelva & 170 & 243 & 189 & 115 & 983 \\
Córdoba & 353 & 443 & 310 & 228 & 1642 \\
Jaén & 256 & 252 & 242 & 153 & 1073 \\
Almería & 189 & 175 & 100 & 108 & 680 \\
\hline Total & $2.772(27 \%)$ & $2575(25 \%)$ & $2.280(22 \%)$ & 1.298 & 10.471 \\
& & & & $(13 \%)$ & \\
\hline
\end{tabular}

Elaboración propia a partir de la información obtenida en Internet:

http://www.juntadeandalucia.es/empleo/macenco/avanzada.jsp

Se trae a colación, para confrontar con esta realidad de los centros colaboradores, que para Billett y Seddon (2004, p. 58-59) los gobiernos actuales están más interesados en facilitar la coordinación entre instituciones, y potenciar las capacidades de individuos y organizaciones, entre las que participan también entidades locales. Estos autores siguen la definición del término "capacidad" que recoge las Naciones Unidas, concebida como la posibilidad que tienen los individuos y las organizaciones para desarrollar actividades con eficacia y eficiencia. Vista la controvertida concentración de centros en pocas especialidades formativas en toda Andalucía, y los ejemplos de los centros colaboradores de Osuna y Estepa en cuanto a los criterios que marcan sus especialidades homologadas, se deduce de ello que los centros colaboradores tienden a estar más guiados por principios pragmáticos, que responden a los intereses inmediatos del centro solicitante de la homologación. Esto hace que el propósito de enmienda que apuntaban Billett y Seddon, en la línea de capacitar a individuos y organizaciones, sea tan necesario para conseguir de forma eficaz y eficiente los objetivos propuestos, y que de este modo la política de formación para el empleo se desarrolle de forma responsable y comprometida con la comunidad. 
En cuanto a la tipología de desempleados/as de esta modalidad formativa, viene marcada por criterios sociales referentes al sexo, edad, discapacidad y otras situaciones sociales de exclusión ${ }^{12}$. Según la información recabada en los monográficos consultados de 2005 y 2009 , un $66 \%$ de los cursos realizados en Andalucía fueron dirigidos a desempleados en general, un $7 \%$ fue específico para mujeres, un 2,5\% para discapacitados, y un 1,5\% para minorías étnicas u otros colectivos desfavorecidos. En el conjunto andaluz, y para 2005, el $61 \%$ del alumnado en estos cursos fueron mujeres, descendiendo la participación femenina al $55 \%$ en 2009, en plena crisis económica. Al confrontar con las características del alumnado de estos cursos en Osuna y Estepa en el periodo 2003 a 2009, se observa similitud a este respecto, por la alta participación de mujeres en el total de cursos impartidos, que llega a ser en Osuna del $73 \%$ y en Estepa del 67\%. Si se compara con otros colectivos si se aprecian diferencias, por ejemplo, en Estepa no se organizó ningún curso específico para personas discapacitadas en ese periodo, en tanto que en Osuna un $7 \%$ de los cursos fueron específicos para este colectivo, impartidos por la asociación de discapacitados físicos de esta localidad, y bajo la supervisión de la Confederación Andaluza de discapacitados ${ }^{13}$. En general, estas prácticas asociadas a la clasificación ${ }^{14}$ de los parados/as moldean la forma en la cual se piensa el problema del desempleo. La clasificación es relevante para la posible acción que toma la institución, ya que es una poderosa herramienta de identificación social, que guía no sólo a los individuos, sino a la forma colectiva de pensar y actuar ${ }^{15}$, y en el contexto de las organizaciones internacionales busca reducir la complejidad (Caswell, et al., 2010, p.385).

En la actualidad, la construcción de identidad centrada en el trabajo se debilita, y se da "una explosión de identidades que coinciden con los supuestos básicos del discursos postmoderno: el recursos al disenso, la discontinuidad, la heterogeneidad" (Alonso, 2007, p.190). Cada vez en mayor medida las demandas formativas parten de los servicios de orientación al empleo, basándose en los itinerarios formativos ${ }^{16}$ que diseñan los orientadores laborales junto con la persona desempleada. Es el técnico de los servicios de empleo el que establece los objetivos profesionales del desempleado en base al diagnóstico de sus capacidades y de sus necesidades formativas (Serrano, Fernández y Artiaga, 2012, p.52). En línea con esto, y siguiendo las reflexiones de Rosanvallon (2012, p.127), se ha pasado de una 


\section{Carvajal Muñoz - Formación para el Empleo}

sociedad preocupada por la redistribución social, bajo los principios de la sociedad salarial (periodo keynesiano), a concepciones más individualistas para justificar la desigualdad que van unidas a transformaciones del capitalismo y de la sociedad, y en este sentido habla del traspaso de un individualismo de la universalidad, propio del periodo anterior, a un individualismo de la singularidad. Este cambio se reflejaría en la tendencia actual a establecer itinerarios formativos personalizados acordados entre la persona desempleada y el técnico de orientación al empleo.

Pero además, conviene tener en cuenta que la formación para el empleo se compromete también en educar en competencias generales, como recoge la normativa $^{17}$, reconociendo la necesidad de dedicar unas horas a la formación transversal de igualdad de género, prevención y protección contra la violencia de género, fomento de la formación a lo largo de la vida, relacionada con el ámbito laboral, pero también con objeto de satisfacer la realización personal y social. Por tanto, no hay que perder de vista que la política de formación para el empleo, como la educación en general, contribuyen a un efecto social multiplicador de competencias de la gente excluida socialmente, pero también de las organizaciones o instituciones participantes (Nicaise, 2012, p.338).

\section{Alumnado y Entidades Locales Participantes en las Escuelas Taller, Casas de Oficios y Talleres de Empleo}

Las Escuelas Taller y Casas de Oficios iniciaron su andadura por las mismas fechas que el programa de cursos de formación visto en la sección anterior (1985), y dirigidas a combatir el desempleo juvenil ${ }^{18}$, gestionándola la administración central hasta 2003, año en el que se da el traspaso de competencias a la comunidad autónoma andaluza ${ }^{19}$. Cuatro años antes, en febrero de 1999, el gobierno central ya había aprobado los Talleres de Empleo $^{20}$, encaminados a formar a parados de 25 o más años, con especiales dificultades de inserción laboral, dando preferencia a los colectivos más vulnerables $^{21}$ según los Planes Nacionales de Acción para el Empleo de cada año. Uno de los objetivos principales de esta formación es mejorar las condiciones laborales de los desempleados/as en peores circunstancias sociales, posibilitando la creación de empleos estables y de calidad, y reduciendo asimismo la precariedad laboral. 
El desempleo es un problema de tal embargadora para cualquier gobierno, que la política de formación se convierte en un importante instrumento para darle respuesta. Si bien en los inicios de este programa, en la década de los ochenta, el desempleo juvenil era el problema más acuciante, también afecta a desempleados/as de otros intervalos de edad. Según la EPA, en diciembre de 2014 el desempleo de mayores de 25 años llegó al 32\% en Andalucía, aunque el paro entre los menores de 25 años fue todavía más alto, alcanzando el $59 \%$ en el último trimestre de 2014. Justamente Aluja (2005, p.192) justifica la aprobación de los Talleres de Empleo en 1999 por la prioridad dada a las mujeres en las directrices del Plan Nacional de Acción para el Empleo (PNAE) aprobado por el gobierno en 1999, dada la alta tasa de paro femenina en esa fecha. Siguiendo con Aluja, esto ha llevado también a una clara feminización de este programa formativo, hasta el punto de que el 60\% del alumnado de TE está representado por mujeres. De hecho, como indica el gráfico de abajo, hay un incremento notable de este programa formativo de 1999 a 2002 en España. Pero aún es mayor el número de TE en 2010, según recoge la tabla 3 , ya que a todas las provincias andaluzas se les aprobaron principalmente proyectos de Talleres de Empleo en este año, en detrimento de las Escuelas Taller y Casas de Oficios, que se han reducido paulatinamente desde 1999. 


\section{ESCUELAS TALLER, CASAS DE OFICIOS Y TALLERES DE EMPLEO EN ESPAÑA}

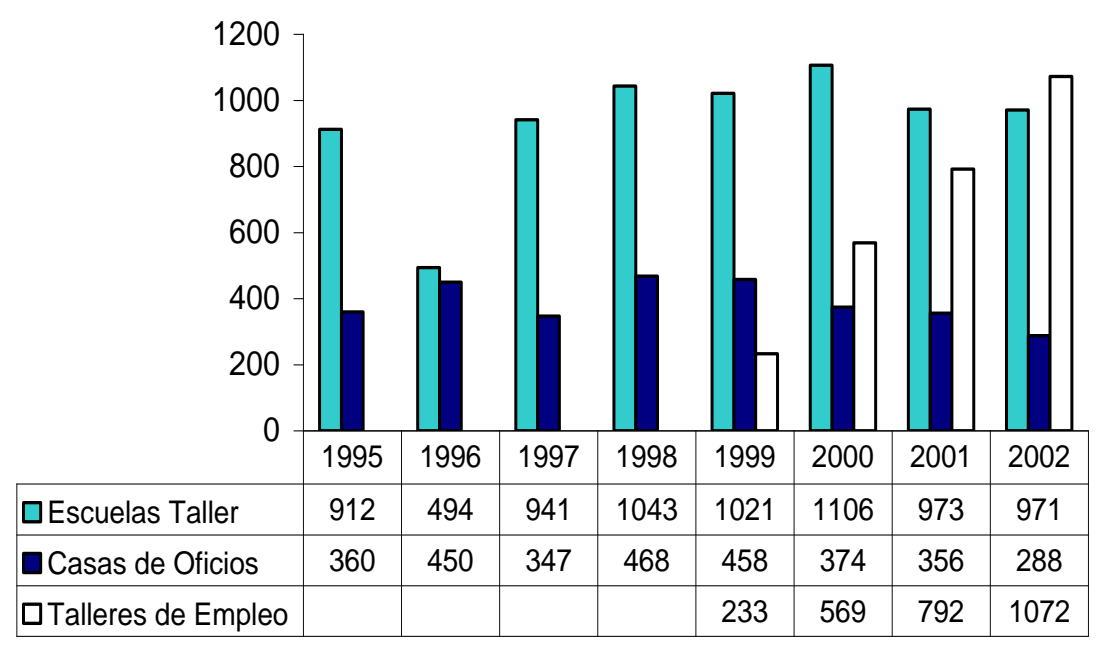

Figura 1. Escuelas Taller, Casas de Oficios y Talleres de Empleo en España

La activación al empleo, implícita en estas acciones formativas, implica el propósito de hacer al individuo más participativo, para que sea capaz de autogobernarse y de manejar sus propios riesgos y recursos, por lo que se le da preferencia a las personas desempleadas en situación de exclusión social (Marston and McDonald, 2005, p.381). De hecho, esta modalidad formativa también comprende módulos transversales que educan al desempleado/a en cuestiones de índole laboral, pero también en un ámbito educativo más general. En el ámbito laboral estos programas contemplan módulos formativos para el fomento de la actividad empresarial y de prevención de riesgos laborales, así como otros módulos referidos a cuestiones de educación general que favorezcan la convivencia con el entorno natural y comunitario, en concreto el módulo de sensibilización medioambiental, el de igualdad de género, y de igualdad para colectivos desfavorecidos, cada uno de ello de diez horas de duración ${ }^{22}$. Estos módulos constituyen una formación transversal que pretende educar en valores de respeto a los sectores sociales más desfavorecidos, teniendo una directa relación con el 
hecho de que las políticas de empleo enfatizan la vinculación existente entre empleabilidad y educabilidad (Spinosa, 2007). La empleabilidad reconoce en esta formación un instrumento para facilitar la inserción laboral, en cambio, el término educabilidad (va unido al principio de equidad, Spinosa, 2007, p.247), y enlaza con el concepto de resiliencia ${ }^{23}$. La educabilidad se asienta justamente en el interés que toman estas políticas por lo relacional, cobrando un valor implícito en sí mismo (resiliencia), y a pesar de que la responsabilidad de la situación de desempleo sigue recayendo todavía en el individuo parado (Spinosa, 2007, p.251).

Tabla 3

Escuelas Taller, Casas de Oficios y Talleres de Empleo en Andalucía en 2010

\begin{tabular}{lccc}
\hline & Escuelas Taller & Casas de Oficio & Talleres de Empleo \\
\hline Sevilla & 15 & - & 54 \\
Málaga & 16 & 1 & 34 \\
Cádiz & 18 & - & 33 \\
Córdoba & 3 & - & 35 \\
Almería & 1 & 13 & 45 \\
Huelva & 1 & 3 & 21 \\
Jaén & 4 & 1 & 34 \\
Granada & 1 & 2 & 71 \\
Total & 59 & 20 & 329 \\
\hline
\end{tabular}

Elaboración propia a partir de la información obtenida en Internet:

https://www.juntadeandalucia.es/empleo/www/te-formamos/recursos-para-laformacion/centros-de-formacion/

En la tabla 3 puede verse el incremento considerable de Talleres de empleo aprobados en todas las provincias andaluzas en 2010, muy por encima de los de Escuelas Taller y Casas de Oficios. Esto hace que se hayan beneficiado de estos programas más desempleados de 25 o más años que jóvenes menores de esta edad. En cuanto a las entidades promotoras de estos TE hay que destacar la significativa participación, aunque en mucha menor medida que ayuntamientos y otras entidades públicas, de las entidades locales sin ánimo de lucro, como asociaciones de mujeres, de gitanos, de discapacitados, además de organizaciones no gubernamentales.

En Andalucía, del total de proyectos de TE aprobados en 2010, un 4\% correspondieron a los presentados por las federaciones y asociaciones de personas discapacitadas ${ }^{24}$. Participaron principalmente federaciones $y$ 


\section{Carvajal Muñoz - Formación para el Empleo}

asociaciones de discapacitados/as físicos, seguidos de la federación andaluza de asociaciones de discapacitados/as auditivos. Este interés por la inserción laboral de las personas discapacitadas, que también se aprecia en los cursos de formación vistos en la sección anterior, coincide con la observación de Malo (2003, p.99), quién subraya que en los últimos años en España cobra especial relevancia la situación laboral de las personas discapacitadas. Esto hace que el gobierno preste mayor atención en atender también las demandas formativas de este colectivo como medio para favorecer su inserción laboral $^{25}$. Según un estudio realizado sobre la política de inserción ofertadas a este sector de la población en Bélgica, se reconoce un debilitamiento de las políticas del estado del bienestar (welfare), constatando los autores que este tipo de políticas de activación al empleo se da también en otros países como Gran Bretaña, con recortes en sus ayudas asistenciales en tanto se les promueve en la búsqueda de empleo (workfare), situación que definen de “incitación al empleo de los discapacitados/as" (Roets et al., 2011, p.2). Hay que destacar con respecto a las políticas de activación al empleo, y entre estas la política de formación para el empleo, que la participación en la formación implica que la persona discapacitada se relacione con sus iguales, compartiendo intereses y preocupaciones (Lawy and Biesta, 2006). Es cierto que los programas de formación de Escuelas Taller, Casas de Oficios y Talleres de Empleo priorizan el trabajo pagado, "activación al empleo que recuerda a sus participantes discapacitados, no tanto la estricta obligación de trabajar, como sí el derecho al empleo" (Roets et al., 2011, p.11). No obstante, se insiste en que es importante considerar otras matizaciones en torno a la participación de las personas desempleadas, y de los agentes locales implicados en esta formación, y que apuntan a la necesidad de analizar y profundizar en las consecuencias que tiene la participación en las acciones formativas para la vida cotidiana de la persona discapacitada, y para la representatividad e intereses de los agentes locales participantes, no necesariamente vinculando la participación con el único propósito de la inserción laboral.

En lo que respecta a las áreas formativas, partiendo de la información recabada de los proyectos aprobados en 2010 en Andalucía, se distinguen particularmente seis áreas formativas ${ }^{26}$. Comparando las especialidades formativas de los proyectos desarrollados en las ocho provincias andaluzas se comprueba que no existe una diferencia significativa entre las 
especialidades ofertadas desarrolladas en cada una de ellas. Muy al contrario, se observa una repetición de las mismas especialidades formativas en todas y cada una de estas provincias, coincidiendo las ocho provincias en las mismas reiteradas actividades profesionales ${ }^{27}$. Esto tiene mucho que ver con la forma en la que se ha diseñado esta formación para el empleo desde arriba, empezando por instancias superiores de la Unión Europea, y corroborada por el gobierno central español. En concreto, se hace referencia a la relación existente entre las áreas formativas de TE, ET y CO con la apuesta hecha por la Comisión Europea por los nuevos yacimientos de empleo para fomento del desarrollo local, partiendo de la promoción del patrimonio cultural y natural y de otros servicios de atención comunitaria. Éstas tienen una relación directa con las áreas formativas de los yacimientos de empleo ${ }^{28}$ que recoge el Libro Blanco de Crecimiento, Competitividad y Empleo publicado en 1994. Incluso antes, la apuesta por el fomento patrimonial como medio para el desarrollo económico ya está implícita en el concepto de patrimonio que recoge la Ley del Patrimonio Histórico Español $^{29}$, ya que define el patrimonio en términos generales, como realidad que incluye el patrimonio documental y bibliográfico, los yacimientos y zonas arqueológicas, así como los sitios naturales, jardines y parques, e insiste en el preámbulo que dicho patrimonio merece de la sensibilidad de los ciudadanos, pues cumple una función social. Por tanto, desde una perspectiva más amplia, el patrimonio se entiende como un bien social, valorándose su promoción en tanto recurso social, económico y cultural (Caravaca, et al., 1997, p.144). En esta misma línea, las ET, CO y TE se ajustan a la nueva concepción del patrimonio que remite a un bien social, económico y cultural. A este respecto, Juara (1993, p.32) recoge justamente cómo la modalidad formativa de ET y CO, regulada en $1988^{30}$, subrayaba entonces el interés de esta formación para la recuperación y promoción del patrimonio cultural y natural, pero también con objeto de que contribuya a la mejora de las condiciones de vida de la comunidad.

A partir de esta definición amplia de patrimonio, concebido como un medio para el fomento del empleo en el entorno comunitario, cabría esperar exitosos logros en la inserción laboral de los formados en estos programas formativos si las premisas teóricas planteadas desde las instancias superiores de la Comisión Europea, y del gobierno central, se hubiesen demostrado efectivas. Pero las evaluaciones realizadas sobre el nivel y calidad de la 


\section{Carvajal Muñoz - Formación para el Empleo}

inserción laboral del alumnado de esta modalidad formativa resultan poco plausibles. Alujas (2005, p.197-198) destaca para España que la tasa de inserción laboral del alumnado masculino supera al femenino, aunque la inserción laboral femenina mejoró en los últimos años, pero en general se trataba de una inserción laboral con alta incidencia de contratos temporales, y en su mayoría referidos a trabajos de especialidades formativas no relacionadas con la formación recibida. En esta línea, De Miguel et al. (2008) realizaron una investigación en Asturias entre 2001 y 2004 utilizando técnicas cuantitativa y cualitativa (encuestas, entrevistas y grupos de discusión) con participantes de 100 proyectos de Talleres de Empleo. El alumnado era en su mayoría mujeres, con edades comprendidas entre 25 a 45 años, y de nivel académico limitado, ya que apenas había finalizado los estudios básicos. Los autores reconocían la necesidad de mejoras significativas en la implementación de este programa para favorecer la inserción laboral. En cuanto al grado de satisfacción del alumnado, Suárez (2004, p.309) realizó una investigación utilizando la técnica de la encuesta y entrevista en profundidad con alumnas de Talleres de Empleo en Sevilla, constatando su alta satisfacción por la formación recibida, pero también destacaba la preocupación que expresaron por la inserción laboral, por lo que demandaban la adopción de medidas que mejorasen las opciones de inserción una vez terminada la formación.

Tampoco hay que perder de vista que esta modalidad formativa de ET, CO y TE se encuadra dentro de la promoción que se hace por la educación permanente $^{31}$, y como tal impulsora del desarrollo social y personal (Sotés, 2005, p.173). En cierto modo, se busca que la formación contribuya a "la educación integral de la persona", como se comentó antes al referir sobre los módulos formativos transversales de estos programas, junto con el "desarrollo del entorno comunitario" (Cabello, 2002, p.185-186). Esta combinación de formación y educación va pareja a la participación de instituciones diversas de la sociedad civil en la implementación de estos programas formativos. En 2010, la participación en esta modalidad formativa de entidades locales de cariz social, como asociaciones, federaciones y organizaciones no gubernamentales, representaron un $6 \%$ del total de los proyectos; las fundaciones, consorcios e institutos un 7,5\%, las Diputaciones un 5\%, y los Organismos Autónomos Locales ${ }^{32}$, pertenecientes al gobierno municipal, otro 5\%. En su conjunto, la corporación municipal 
acaparó el 79,4\%, incluyendo en esta suma el porcentaje anterior de los Organismo Autónomo Local (5\%), junto con el 57,5\% de proyectos aprobados a los ayuntamientos, y el 17,2\% aprobado a las mancomunidades. Esto indica que el grueso mayor de los proyectos aprobados, casi el $60 \%$, fueron para ayuntamientos, seguidos de las Mancomunidades. Por tanto, al comparar la participación del consistorio con la de los agentes locales de la sociedad civil se constata que estos últimos, aún siendo muy significativa su participación, es escasa con respecto al protagonismo dado al gobierno municipal en esta modalidad formativa de ET, CO y TE.

\section{Conclusiones y Consideraciones Finales}

Sintetizando los resultados más destacados del estudio, entre la participación de los agentes locales se constata el alto protagonismo de la corporación municipal en la realización de los proyectos de ET, CO y TE. En los casos particulares de Osuna y Estepa también se confirma una alta participación de los ayuntamientos y de empresas públicas en la realización de cursos de formación, en cambio, en el conjunto andaluz la participación de las corporaciones municipales en la implementación de estos cursos sólo supone un $13 \%$, frente al $47 \%$ de entidades sin ánimo de lucro. A esto se le une que la tipología de las especialidades formativas que homologan los centros colaboradores para sus cursos de formación está muy determinada por las características de la entidad ${ }^{33}$, pero también lo está por las directrices que se marcan desde arriba, desde instancias superiores ${ }^{34}$. En no pocas ocasiones se tienen en cuenta también las demandas formativas de los desempleados, caso de los ayuntamientos particularmente. La determinación de las instancias superiores está claramente presente en las especialidades formativas de los programas de ET, $\mathrm{CO}$ y TE, ya que han delimitado sus áreas formativas en temas específicos en torno al patrimonio y los servicios a la comunidad, según recoge su normativa.

En cuanto a la participación de los desempleados/as se confirma la presencia de colectivos muy heterogéneos, aunque en la modalidad de cursos de formación se impartieron principalmente para desempleados/as en general, siendo muy reducido el número de cursos específicos para mujeres, para discapacitados, u otros colectivos en riesgo de exclusión. La misma heterogeneidad se da también entre el alumnado de ET, CO y TE. No 


\section{Carvajal Muñoz - Formación para el Empleo}

obstante, en los últimos años, a partir de 1999, se incrementa considerablemente el número de TE en detrimento de las ET y CO, por lo que hay una mayor participación de desempleados/as de 25 o más, preferentemente mujeres, que supera el $50 \%$ del total del alumnados. Asimismo, se constata un porcentaje muy pequeño, pero significativo, de proyectos de TE para colectivos de discapacitados en todas las provincias andaluzas en 2010. Por último, hay que destacar la tendencia que está tomando la formación para el empleo adecuándose a las particularidades de cada desempleado/a en base a los itinerarios formativos. Tanto la clasificación que se hace de la población desempleada, basada en criterios referidos a edad, género, vulnerabilidad social, como los objetivos que se marcan estas acciones formativas según normativa, indicando la construcción social de identidades que van parejas a una nueva organización sociopolítica, diferente a la que se dio en la sociedad salarial.

Siguiendo a Di Domenico et al. (2010, p.687), sería necesario pensar en programas de intervención frente al problema del desempleo que sean eficaces a la realidad concreta de cada territorio, evitando las teorizaciones o planteamientos abstractos. Es cierto que las entidades locales se han adaptado a los criterios que marcan estas modalidades formativas de un modo bastante pragmático, hasta donde los programas les permiten, e incluso podría decirse que se han adaptado con sentido práctico y marcadamente individualista. Esta realidad va unida al hecho de que en lo que se refiere a los objetivos teóricos que recogen las normativas, entre los que destaca el favorecer la inserción laboral de las personas desempleadas, los resultados son bastante insatisfactorios. En parte, por haberse diseñado desde instancias superiores (organismos internacionales y de ahí a los Estados), de una forma general y teórica; pero también por las particularidades propias de las entidades locales existentes en cada territorio. En este sentido, falta un basamento más sólido en el diseño de la política de formación adaptable a las peculiaridades de los mercados de trabajos, y a la vida relacional en sí, propiciando una mayor y mejor interacción entre las entidades locales que ofrecen formación, y de éstas con los desempleados/as y con los agentes del tejido empresarial local y autonómico. Este rediseño de la política de formación debe completarse con otras actuaciones mediante las que sea posible, de un modo eficaz y eficiente, la inserción laboral, y/o contribuyan a mejorar realmente la convivencia en la comunidad (Moral, 


\section{Notas}

${ }^{1}$ La Orden de 31 de julio de 1985 del Plan Nacional de Formación e Inserción Profesional regulada los cursos de Formación Profesional Ocupacional a partir de los ochenta, y estaban impartidos por centros colaboradores del INEM (Instituto Nacional de Empleo). La modalidad formativa de los programas de TE y CO funcionan desde 1985 combinando formación teórica y práctica. Ambos programas van dirigidos a personas desempleadas menores de 25 años. Los TE tienen una duración máxima de 2 años y las CO de 1 año. La formación se imparte en alternancia con el trabajo o práctica profesional, y se da prioridad a los proyectos cuyas características fundamentales sean la participación activa de los jóvenes y su relación inmediata con el entorno comunitario (Art. 4, de la Orden de 29 de marzo de 1988). Los TE funcionan desde 1999, también combinan un periodo formativo con la práctica profesional, van dirigidos a personas desempleadas de 25 o más años, con una duración mínima de seis meses y máxima de 1 año (Art. 1-3 del Real Decreto 282/1999 de 22 de febrero que establece la aprobación de los TE).

${ }^{2}$ Se realizaron entrevistas en profundidad a monitores de cursos, alcaldes de los dos municipios, técnicos de formación de los ayuntamientos en estas dos localidades, a los responsables en la delegación en Sevilla, y a los representantes de las entidades locales que impartieron entre 2003 a 2009 estas acciones formativas (Carvajal, 2002, 2010, 2014). ${ }^{3}$ Real Decreto 427/1993 de 26 de marzo sobre la transferencia de competencia de este programa formativo de la administración central a la comunidad autónoma andaluza.

${ }^{4}$ Pueden ser centros colaboradores las entidades locales, públicas o privadas, con o sin ánimo de lucro, que reunan una serie de requisitos mínimos de condiciones higiénicas, acústicas, de habitabilidad y de seguridad, de espacios disponibles y que dispongan de profesorado adecuado (Real Decreto 631/1993).

${ }^{5}$ La crisis económica comprendida en el periodo que va de 2011 a 2015, afecta a la oferta de cursos y número de alumnados beneficiados, reduciéndose en 1285 los cursos, y en 17178 alumnos/as menos en 2009 con respecto a 2005, según los monográficos consultados de 2005 y 2009, y publicados por la Junta de Andalucía.

${ }^{6}$ La información detallada sobre estos monográficos viene en la bibliografía. Pero conviene referir que se tratan de pequeños cuadernillos, a modo de informes anuales muy descriptivos sobre la marcha de este programa en Andalucía, distinguiendo entre tipología de entidades locales, colectivos de desempleados, diferencias entre provincias, entre otras cuestiones.

${ }^{7}$ Las corporaciones locales realizaron un $13 \%$ del total de los cursos, las entidades privadas un $30 \%$, las entidades públicas un $10 \%$, y las entidades sin ánimo de lucro un $47 \%$. Hay que tener en cuenta que entre las entidades sin ánimo de lucro se incluyen también asociaciones que están bajo la responsabilidad de los ayuntamientos, con lo que se ampliaría la competencia de las corporaciones municipales en estas acciones formativas para el conjunto andaluz.

${ }^{8}$ De los datos recabados en la Consejería de empleo sobre los centros colaboradores de los municipios de Osuna y Estepa sobre los cursos y centros que los impartieron en el periodo de 2003 a 2009, se confirma que el ayuntamiento de Estepa realizó un 40\% de los cursos para este periodo. El ayuntamiento de Osuna algo menos, un $25 \%$ del total de los que se 


\section{Carvajal Muñoz - Formación para el Empleo}

impartieron. Pero ambos municipios disponían (y disponen) de organismos públicos, como Mancomunidades y otras instituciones en Estepa, y la Fundación Pública Francisco Maldonado de Estudios Universitarios, que hacen que el porcentaje de cursos impartidos bajo el patrocinio de la corporación municipal se eleve considerablemente, superando el $60 \%$ del total de cursos para cada caso.

${ }^{9}$ Evidentemente, la normativa de cualificación profesional de la formación para el empleo contempla otras áreas formativas, en concreto, en la página Web consultada para la elaboración de la tabla 2 se registran hasta 27 áreas formativas, pero aquí sólo se destacan las más significativas por ser las áreas formativas que más centros colaboradores han homologado en Andalucía.

${ }^{10}$ Información obtenida de: http://www.juntadeandalucia.es/empleo/macenco/avanzada.jsp

${ }^{11}$ En 2012 el 11\% de los centros colaboradores estaban homologados en esta especialidad formativa, en 2015 llega al $13 \%$.

${ }^{12}$ Los monográficos de la Junta de Andalucía clasifican a la población desempleada en desempleados en general, en colectivos de mujeres, de jóvenes, de discapacitados, colectivos pertenecientes a minorías étnicas u otros grupos desfavorecidos, a los privados de libertad, y a los colectivos de inmigrantes. Esta clasificación de los desempleados es bien distinta a la que se comentó antes en la que se incluía una atención preferente también para atender a necesidades formativas debidas a la reconversión industrial, teniendo esta una relación mucho más directa con el ámbito laboral.

${ }^{13}$ En el trabajo de campo realizado en estos municipios también se comprueba la interacción existente entre asociaciones y sindicatos con sus superiores jerárquicos. Así, las asociaciones de discapacitados dependen en buena medida de la Confederación, aunque también cuentan con el respaldo del ayuntamiento. Los sindicatos están supeditados principalmente a las directrices de la federación provincial. Las demás asociaciones necesitan de la colaboración también del ayuntamiento, que les facilita el local, y en no pocas ocasiones les asesora en qué acciones formativas solicitar a la Junta. Esta panorámica subraya cómo existe interdependencia o interpenetración entre la sociedad civil y el Estado, que ya ha sido estudiada en otros países (Maloney, et al, 2000).

${ }^{14}$ Sobre el poder que tiene clasificar a la población para la construcción social de la realidad véase también Douglas (1996), Hacking (1985), Foucault (2005).

${ }^{15}$ Los discursos que constituyen esta política de formación para el empleo tiene mucho que ver con cuestiones importantes en torno a temas centrales relativos a la crisis de la sociedad salarial (Carvajal, 2010:70).

${ }^{16}$ El Decreto 335/2009 de 22 de septiembre, en su Art. 3, distingue entre itinerario formativo, programa formativo, proyecto formativo y plan de formación, muy encaminados a adecuar la formación a las características individuales de los demandantes de empleo.

${ }^{17}$ Decreto 225/2009 de 22 septiembre de integración de la Formación Profesional Ocupacional y la Formación continúa.. En capítulo 1, disposiciones generales, artículo 2.

${ }^{18}$ Como recoge la Orden Ministerial de 29 de marzo de 1988 que regulaba esta modalidad de formación para el empleo, se beneficiaban de estas acciones formativas los jóvenes menores de 25 años con dificultades de inserción laboral.

${ }^{19} \mathrm{El}$ traspaso de esta competencia formativa a la Junta de Andalucía se da por Real Decreto $467 / 2003$ de 25 de abril.

${ }^{20}$ Los Talleres de Empleo se aprueban por Real Decreto 282/1999, de 22 de febrero.

${ }^{21}$ Según la Orden 21 de noviembre de 2008, el programa formativo da preferencia a la formación para colectivos con especiales dificultades de inserción laboral, como mujeres, jóvenes menores de 25 años, parados de larga duración, demandantes en riesgos de exclusión, 
personas con discapacidad, minorías étnicas e inmigrantes legalmente documentados, entre otros

${ }^{22}$ Se recoge en el artículo 6 de la Orden de 5 de diciembre de 2006 que se regulan los programas de Escuelas Taller, Casas de Oficios y Talleres de Empleo.

${ }^{23}$ Resiliencia entendida como el resultado de un proceso adaptativo de las personas en interacción con su entorno que les lleva a desarrollarse de forma más sana (Rutter, 1993).

${ }^{24}$ En concreto, les aprobaron Talleres de Empleo para colectivos con discapacidad a las asociaciones y federaciones de discapacitados/as de las provincias de Sevilla, Málaga, Almería, Jaén, Granada y Cádiz, en 2010, destacando la federación andaluza de personas con discapacidad física en Sevilla y Málaga, la federación andaluza de personas sordas en Sevilla y Jaén, Aspaym (asociación de discapacitados medulares y de grandes discapacidades físicas) en Granada, El saliente (Asociación de personas con discapacidad) en Almería, Amivel (Asociación Minusválidos Veleños y de la Axarquía) en Málaga, y la Asociación Provincial de Familiares de Personas con Trastorno de Espectro Autista en Cádiz.

${ }^{25}$ Caswell et al. (2010:386) también refieren el caso de Australia, donde algunos colectivos de discapacitados, catalogados antes como no válidos para trabajar, se les etiqueta a partir de 2006 como válidos para el trabajo. Asimismo, en Dinamarca, a partir de 2003 grupos de discapacitados/as con derecho a asistencia social vieron reducidos estos derechos en tanto se les incentivaba al empleo, entrando así en la dinámica de las políticas de activación.

${ }^{26}$ Las seis áreas formativas desarrolladas principalmente en los Talleres de Empleo son: 1. Servicios socioculturales y a la comunidad; 2 . Servicios de atención al entorno natural; 3 . Servicios turísticos; 4. Servicios patrimoniales; 5. Oficios tradicionales; y por último, 6. Instalador de equipos de energía renovable.

${ }^{27}$ En particular, destacan las siguientes actividades laborales en Talleres de Empleo de estas provincias andaluzas: Animación sociocultural, atención a discapacitados, atención a la infancia, ayuda a domicilio, cultivo de plantas aromáticas, monitor de espacios naturales, agente de turismo rural, de turismo de aventura, agente de desarrollo turístico, restauración de patrimonio, además de actividades en oficios tradicionales como: elaboración de conserva, licores y pasteles, ebanistería, mampostería, apicultura, guarnicionería, marroquinería, entre otros oficios de la misma índole.

${ }^{28}$ La referencia a los nuevos yacimientos de empleo viene recogida en el preámbulo del Real Decreto 281/1999 que aprueban los TE, en el que se reconoce literalmente que "este programa de formación y empleo llevará a cabo actividades relacionadas con los nuevos yacimientos de empleo en interés general y social, promovidas por entidades públicas o privadas sin ánimo de lucro". En cuanto a la modalidad formativa de cursos de formación, el Decreto 204/1997 de 3 septiembre que establece los programas de Formación Profesional Ocupacional de la Junta de Andalucía reconoce en su Art. 4.7, entre las especialidades homologables a los centros colaboradores la de los nuevos yacimientos de empleo (servicios de utilidad colectiva, servicios de ocio y culturales, servicios personalizados de carácter cotidiano).

${ }^{29}$ Ley $16 / 1985$ de 25 junio.

${ }^{30}$ En Orden Ministerial 1988 de 29 de marzo que ofrece una primera regulación de las Escuelas Taller y Casas de Oficios.

${ }^{31}$ Hay muchos ejemplos referido a normativas que insisten en la formación a lo largo de la vida como una característica ya consustancial a la formación para el empleo. Por hacer referencia a alguna de estas, el Decreto 225/2009 de 22 de septiembre que integra la formación profesional ocupacional, que recoge la sección anterior de este trabajo, con la formación continua, llamándose ahora Formación Profesional para el Empleo. Esta normativa 


\section{Carvajal Muñoz - Formación para el Empleo}

hace referencia expresa a la necesidad de fomentar la formación a lo largo de la vida "en adaptación permanente de la población activa a las cualificaciones demandadas en el entorno laboral y permitiéndoles su realización personal y social".

${ }^{32}$ Los Organismos Autónomos Locales están regulados por la Ley 57/2003 de 16 de diciembre y dependen de los gobiernos municipales.

${ }^{33}$ Así, las empresas privadas se homologan en las especialidades formativas que permiten sus instalaciones y su personal cualificado, ya se trate de una academia de informática o una autoescuela éstas homologarán con relación a su perfil profesional. Lo mismo hacen los centros educativos en general que se homologan para funcionar como centro colaborador. ${ }^{34}$ El Real Decreto 34/2008 de 18 de enero, que regula los certificados profesionales, los definen como instrumentos de acreditación oficial bajo la adecuación a las Cualificaciones Profesionales del Catálogo Nacional de cualificación Profesional.

\section{Referencias}

Alonso Benito, L. (2007). Crisis de la ciudadanía laboral. Barcelona: Anthropos.

Aluja Ruiz, J.A. (2005). Las políticas de formación/empleo: Medida singular del eje de formación de las políticas activas de mercado de trabajo en España. Trabajo: Revista Andaluza de Relaciones Laborales, 16, 189-208.

Billett, S., Seddon, T., (2004). Building Community through Social Partnership around Vocational Education and Training. Journal of Vocational Education and Training. 56(1), 51-68.

Cabello Martínez, M.J. (2002). Educación permanente y Educación Social. Controversias y compromisos. Málaga: Aljibe.

Cansino Muñoz-Repiso, J.M., Sánchez Braza, A. (2009). Evaluación del programa de escuelas taller y casas de oficio a partir de su efecto sobre el tiempo de búsqueda del primer empleo. El caso de Sevilla. Estudios de Economía Aplicada, 27(1), 1-22.

Carabaca Barroso, I., Colorado Campos, D., Fernández Salinas, V. Paneque Salgado, P., Puente Asuero, R., Romero Moragas, C. (1997). Patrimonio cultural, territorio y políticas públicas. El caso de Andalucía. Estudios Regionales, 47, 143-160.

Carvajal Muñoz, M.R. (2014). La política de formación para desempleados/as en el entorno local y sus implicaciones sociales y políticas. Margen: Revista de Trabajo Social, 74, 1-19. 
Carvajal Muñoz, M.R. (2012). La relación micro macro en la política de formación para el empleo. Una aportación teórica y metodológica. Nómadas. Revista Crítica de Ciencias Sociales y Jurídicas, 34(2), 119.

Carvajal Muñoz, M.R. (2010). La formación para el empleo bajo una perspectiva foucaultiana. RASE. Revista de la Asociación de Sociología de la Educación, 3(1), 66-83.

Carvajal Muñoz, M.R. (2002). Los cursos de formación ocupacional para desempleados en cuatro municipios de la comarca sierra sur sevillana: ¿Formación para la inserción laboral o intercambio de intereses múltiples entre las organizaciones participantes? Témpora, 5, 103-123.

Caswell, D., Marston, G., Elm, J., (2010). Unemployed citizen or "at risk" client? Classification systems and employment services in Denmark and Australia. Critical Social Policy, 30(3), 384-404.

De Miguel Díaz, M., Pereira González, M., Pascual Diez, J., Carrio Fernández, E.M. (2008). Propuestas para la mejora de las Escuelas taller, Casas de Oficios y Talleres de empleo. Revista Española de Orientación y Psicología Pedagógica, 19(3), 316-327.

Delfino, A. (2011). Marienthal ¿allá lejos y hace tiempo? Potencialidades y límites de los conceptos fundantes de la Sociología de la desocupación para los estudios latinoamericanos actuales. Fermentum. Revista Venezolana de Sociología y Antropología, 60, 11-34.

Di Domenico, M.L., Haugh, H., Tracey, P. (2010). Social Bricolage: Theorizing Social Value Creation in Social Enterprise. Entrepreneurship Theory and Practice, 34(4), 681-703.

Douglas, M. (1996). Cómo piensan las instituciones. Madrid: Alianza Editorial.

Finn, D. (2000). Welfare to Work: The local dimension. Journal of European Social Policy, 10(1), 43-57.

Foucault, M. (2005). La hermenéutica del sujeto. Madrid: Akal.

Juara, C. (1993). Escuelas Taller: la creación de empleo desde la recuperación del patrimonio. Colección informes, $\mathrm{n}^{\circ}$ 19. Madrid: Centro de Publicaciones Ministerio de Trabajo y Seguridad social. 


\section{Carvajal Muñoz - Formación para el Empleo}

Hacking, I. (1985). Making up people, in Heller, T., Sosna, M., and Wellbery, D. Reconstructing Individualism. Stanford: Stanford University Press.

Hutchinson, J., Campbel, M., (1998). Working in Partnership: Lessons from the Literature. DFEE, Research Report, no 69. London: Department for Education and Employment.

Lawy, R., Biesta, G. (2006). Citizenship-as-practice: the educational implications of an inclusive and relational understanding of citizenship. British Journal of Educational Studies, 54, 34-50.

Libro Blanco de Crecimiento, Competitividad y Empleo. Retos y Pistas para entrar en el siglo XXI, (1994). Bruselas: Comisión Europea.

Malo Ocaña, M.A. (2003). Las personas con discapacidad en el mercado de trabajo español. Revista del Ministerio de Trabajo y Asuntos Sociales, 46, 99- 126.

Maloney, W., Smith, G., Stoker, G. (2000). Social Capital and Urban Governance: Adding a more Contextualized "Top-Down" Perspective, Political Studies, 48(4), 802-820.

Marinetto, M. (2003). Who wants to be an active citizen?: The politics and practice of community involvement. Sociology, 37(1), 103:120.

Marston, G., Mcdonald, C., Wright, S. (2011). The Role of Non-profit organizations in the Mixed Economy of Welfare-to-Work in the UK and Australia. Social Policy and Administration, 45(3), 299-318.

Marston, G., Mcdonald, C. (2005). Workfare as welfare: governing unemployment in the advanced liberal stante. Critical Social Policy, 25(3), 374-401.

Martínez Lucio, M., Skule, S., Kruse, W., Trappmann, V. (2007). Regulating Skill Formation in Europe: German, Norwegian and Spanish Policies on Transferable Skills. European Journal of Industrial Relations. 13(3), 323-340.

Monográfico. Formación Para El Empleo. Estadísticas de alumnos formados en cursos de formación para el empleo. (2009). Sevilla: Consejería de Empleo. Servicio Andaluz de Empleo.

Monográfico. Formación Para El Empleo. Estadísticas de alumnos formados en cursos de formación para el empleo. (2005). Sevilla: Consejería de Empleo. Servicio Andaluz de Empleo. 
Moral Jiménez, M. (2007). Preparación para el trabajo de los jóvenes contemporáneos en una sociedad postindustrial: Trabajo, educación y globalización. Estudios de Educación, 13, 171-194.

Peck, J. (1998). New Labourers: Making a New Deal for the "Workless Class", paper presented at the Annual conference of the Royal Geographical Society/Institute of British Geographers (January). London: Guildford.

Pérez- Díaz, V., Fernández, J.C. (2002). La educación profesional en España. Madrid: Fundación Santillana.

Roets Griet, R., Claes, L., Vandekinderen, C. (2011). Reinventing the employable citizen: a perspective for social work. British Journal of Social Work, 7, 1-17.

Rosanvallon, P. (2012). Reflexiones sobre la igualdad en una era de desigualdades. Estudios Internacionales. Revista del Instituto de Estudios Internacionales de la Universidad de Chile, 171, 119-135.

Rutter, M. (1993). Resilience: Some conceptual consideration. Journal of Adolescent Health, 14(8), 626-631.

Sabán Vera, C. (2009). La educación permanente y la enseñanza por competencias en la Unesco y en la Unión Europea. Madrid: Grupo Editorial Universitario.

Sánchez Esteban, N. (2010). Los programas de Escuelas Taller, Casas de Oficios y Talleres de Empleo en España: un instrumento de formación, una herramienta para la conservación del patrimonio, un mecanismo de intervención social. Boletín $C F+S, 42 / 43$. Simposio Internacional Desarrollo, Ciudad y Sostenibilidad, 70, 453-462.

Sanchis, E. (2003). La experiencia de paro. Política y Sociedad, 40(1), 161183.

Serrano P., Amparo, Fernández Rodríguez, C., Artiaga Leiras, A. (2012). Ingenierías de la subjetividad: el caso de la orientación para el empleo. REIS. Revista Española de Investigación Sociológica, 138, 41-61.

Sotés Elizalde, M.A. (2005). Enseñanza no reglada y capacitación profesional: una visión de la educación como derecho económico, social y cultural". Estudios sobre Educación, 8, 165-192.

Spinosa, M. (2007). Del empleo a la empleabilidad, de la educación a la educabilidad, en Drolas, Ana, Lenguita, Paula, Montes, Juan (eds.). 


\section{Carvajal Muñoz - Formación para el Empleo}

Relaciones de poder y trabajo. Las formas contemporáneas de explotación. Buenos Aires: Poder y Trabajo Editores, pp. 243-255.

Suárez Ortega, M. (2004). Los talleres de empleo como recursos para la formación y la inserción laboral femenina: Estudio de caso. Enseñanza \& Teaching: Revista Interuniversitaria de didáctica, 22, 301-316.

Turok, I.,Webster, D. (1998). The New Deal: Jeopardised by the Geography of Unemployment?. Local Economy, 12(4), 309-327.

Vaquero García, A. (2005). El abandono escolar temprano en España. Programas y acciones para su reducción. Eduga: Revista galega do Ensino, 47, 1442-1464.

\section{Referencias Legislativas}

DECRETO 225/2009 de 22 septiembre de integración de formación profesional ocupacional y de la formación continúa.

DECRETO 335/2009 de 22 septiembre que regula la ordenación de la formación para el empleo en Andalucía.

LEY 57/2003 de 16 diciembre que regula los organismos autónomos locales.

LEY 16/1985 de 25 junio de Patrimonio Histórico Español.

ORDEN de 29 marzo de 1988 que regula los Escuelas Taller y las Casas de Oficios.

ORDEN de 28 de abril de 2011 por la que se aprueba el programa integral de empleo en Andalucía.

ORDEN de 23 de octubre de 2009 por la que se desarrollo el Decreto $335 / 2009$.

ORDEN 56 de 16 de febrero de 2003, por la que se modifica la Orden de 12 de diciembre de 2000, de Convocatoria y Desarrollo de los Programas de Formación Profesional Ocupacional.

ORDEN de 25 de julio de 2000 por el que se regula el procedimiento de autorización administrativa para la actividad como Centro colaborador de Formación Profesional Ocupacional de la Junta de Andalucía. 
ORDEN de 5 diciembre de 2006 que regula los programas de Escuelas Taller, Casas de Oficios y Talleres de Empleo.

ORDEN de 17 de marzo de 1998, de convocatoria y desarrollo de los programas de Formación Profesional Ocupacional.

ORDEN de 31 de julio de 1985 del Plan Nacional de Formación e Inserción Profesional.

REAL DECRETO 427/1993 de 26 de marzo, de la transferencia de la gestión de la formación profesional ocupacional a la comunidad autónoma andaluza.

REAL DECRETO 631/1993 de 3 mayo que regula el plan nacional de formación e inserción profesional.

REAL DECRETO 797/1995 por el que se establece directrices sobre los certificados de profesionalidad y los correspondientes contenidos mínimos de formación profesional ocupacional.

REAL DECRETO 282/1999, de 22 de febrero donde se regulan y aprueba la creación del programa de Talleres de Empleo.

REAL DECRETO 467/2003 de 25 de abril sobre el traspaso de competencia de la comunidad autónoma andaluza de la gestión del Instituto Nacional de Empleo, en el ámbito de trabajo, empleo y formación.

REAL DECRETO 34/2008 de 18 de enero que regula los certificados de profesionalidad.

María Rosario Carvajal Muñozis Professor of Sociology in the Department of Economy at University of Cádiz.

Contact Address: Direct correspondence to María Rosario Carvajal Muñoz at Facultad de Ciencias Sociales y de la Comunicación, Universidad de Cádiz (Campus de Jerez), Avda de la Universidad núm. 4, CP: 11.406, Jerez de la Frontera (Cádiz) - Cádiz. E-mail: rosario.carvajal@uca.es 